\title{
Spatial resolution limits in electron-beam-induced deposition
}

\author{
N. Silvis-Cividjian, C. W. Hagen, a) and P. Kruit \\ Delft University of Technology, Faculty of Applied Sciences, Lorentzweg 1, 2628 CJ Delft, The Netherlands
}

(Received 26 May 2005; accepted 30 August 2005; published online 24 October 2005)

\begin{abstract}
Electron-beam-induced deposition (EBID) is a versatile micro- and nanofabrication technique based on electron-induced dissociation of metal-carrying gas molecules adsorbed on a target. EBID has the advantage of direct deposition of three-dimensional structures on almost any target geometry. This technique has occasionally been used in focused electron-beam instruments, such as scanning electron microscopes, scanning transmission electron microscopes (STEM), or lithography machines. Experiments showed that the EBID spatial resolution, defined as the lateral size of a singular deposited dot or line, always exceeds the diameter of the electron beam. Until recently, no one has been able to fabricate EBID features smaller than 15-20 nm diameter, even if a 2-nm-diam electron-beam writer was used. Because of this, the prediction of EBID resolution is an intriguing problem. In this article, a procedure to theoretically estimate the EBID resolution for a given energetic electron beam, target, and gaseous precursor is described. This procedure offers the most complete approach to the EBID spatial resolution problem. An EBID model was developed based on electron interactions with the solid target and with the gaseous precursor. The spatial resolution of EBID can be influenced by many factors, of which two are quantified: the secondary electrons, suspected by almost all authors working in this field, and the delocalization of inelastic electron scattering, a poorly known effect. The results confirm the major influence played by the secondary electrons on the EBID resolution and show that the role of the delocalization of inelastic electron scattering is negligible. The model predicts that a $0.2-\mathrm{nm}$ electron beam can deposit structures with minimum sizes between 0.2 and $2 \mathrm{~nm}$, instead of the formerly assumed limit of $15-20 \mathrm{~nm}$. The modeling results are compared with recent experimental results in which 1-nm $\mathrm{W}$ dots from a $\mathrm{W}(\mathrm{CO})_{6}$ precursor were written in a $200-\mathrm{kV}$ STEM on a $30-\mathrm{nm}$ SiN membrane. (C) 2005 American Institute of Physics. [DOI: 10.1063/1.2085307]
\end{abstract}

\section{INTRODUCTION}

Electron-beam-induced deposition (EBID) is a technique to create nanostructures by pinning precursor molecules onto a surface with a focused electron beam. Until recently, the smallest structures made with EBID typically were $15-20 \mathrm{~nm}$ wide although the electron beam creating them was as small as $1 \mathrm{~nm}$. We explained this ${ }^{1}$ by analyzing the role of secondary electrons in the process and were subsequently able to break this limit and write sub-5-nm contamination lines and dots by changing the process parameters. ${ }^{2}$ Since then, other authors ${ }^{3-5}$ have also written metal structures as small as $3.5 \mathrm{~nm}$.

In a typical EBID situation, an electron beam is focused on a solid target in a probe of diameter $d$. The target's surface is covered with a layer of gas molecules, continuously delivered through a nozzle. As a result of complex electroninduced processes, these adsorbed precursor molecules dissociate and under the stationary beam a singular dot grows, shaped as a cylindrical shank with a long conical head. This spot exposure response is interesting for the EBID study because its diameter $D$ gives an indication of the resolution of the process. The evaluation of the EBID resolution is thus reduced to a relation between the quantities $d$ and $D$, for a given target and precursor. Until recently, the size of the

\footnotetext{
a) Author to whom correspondence should be addressed; electronic mail: c.w.hagen@tnw.tudelft.nl
}

deposited structures obtained experimentally always exceeded the diameter of the electron beam used to produce them. It has also been observed ${ }^{6,7}$ that $D$ is not constant in time, but follows an evolution as shown in Fig. 1.

In short, the explanation is that a structure, although it may start very small, grows in all directions because the secondary electrons responsible for the growth exit from the sides of the structure. Thus, the width of the structure becomes approximately twice the path length that secondary electrons can travel. Our aim is to develop a theoretical model in order to elucidate the limits of resolution: how small can $D$ be at the very beginning of the deposition process?

Any EBID model deals with three entities that interact with each other: a solid target, the gas precursor molecules adsorbed on this target, and the electron beam together with other electrons it might generate elsewhere during irradiation. Because we are mainly interested in the lateral evolution of the deposited structures, it is absolutely necessary to quantify the vertical (diameter) axis in Fig. 1. We assume that the electrons participating in the EBID process mainly dictate the diameter $D$. Therefore, we will study their interactions with the gas molecules and the solid target in detail. Another group of interactions, between the gas layer and the target surface, including absorption, desorption, and diffu- 


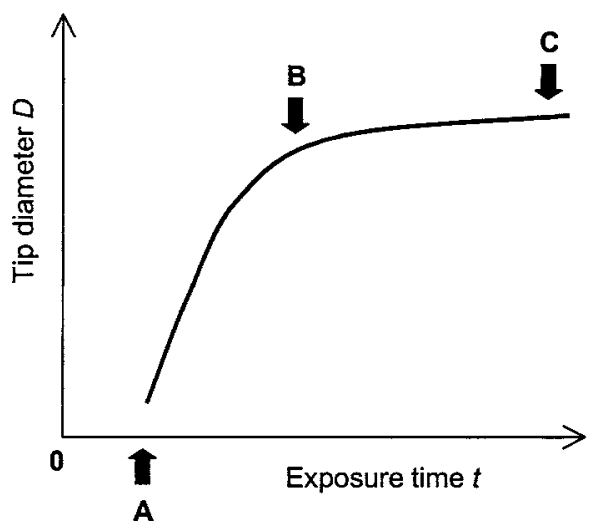

FIG. 1. Experiments show this type of evolution of the EBID spot exposure response, $D(t)$. Three regimes can be distinguished: the nucleation stage (marked 0-A), when no significant growth is observed; an intermediate regime (A-B), characterized by a fast growth of the diameter $D$; and the saturation regime (B-C), where the diameter attains a more or less constant value.

sion is not treated in this article because we expect that they will have more impact on the quantification of the horizontal (time) axis in Fig. 1.

Several types of electrons involved in EBID can be distinguished, ${ }^{8}$ as illustrated in Fig. 2. The primary electrons (PE) are normally incident on the target and focused in a very small spot. Some of the primary electrons can be transmitted through the target, the rest will be backscattered (BSE) and will emerge with almost the same energy on the top surface of the target. Secondary electrons (SEs), generated in the target material as a result of primary irradiation, eventually emerge under different angles on the top surface of the target. We denote the secondary electrons directly generated by the primary electrons by SE1 and the secondary

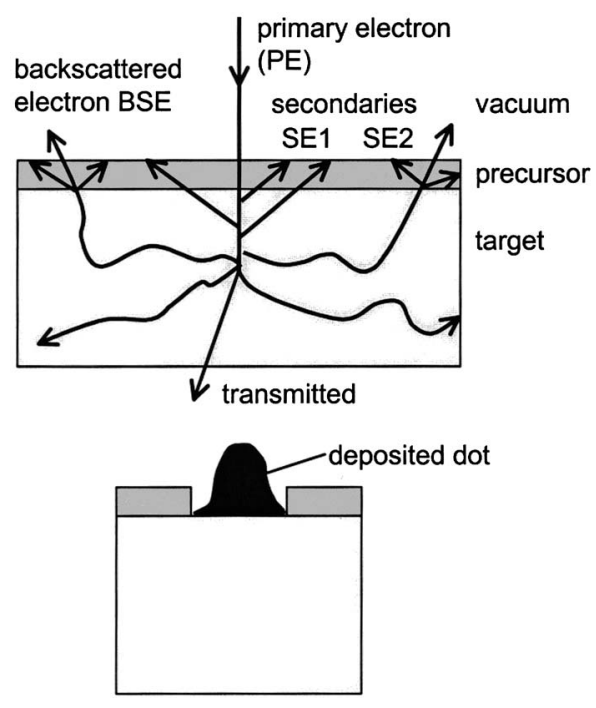

FIG. 2. Illustration of the electron species involved in the EBID model. The primary electrons (PE) irradiate the target covered by precursor molecules. Some electrons, named backscattered electrons (BSE), emerge again from the target top surface. The PE generate the secondary electrons SE1 through inelastic scattering inside the target, while the BSE generate the secondary electrons denoted SE2. As a result of all these electron-induced molecular dissociations of the adsorbed gaseous precursor molecules, a dot is deposited on the target top surface. If the target is thin, a dot will be deposited on its bottom side as well. [from Silvis-Cividjian (see Ref. 8).] electrons generated by the backscattered electrons by SE2. In principle, all these electron species can dissociate adsorbed gas molecules and contribute to the deposition process. However, most EBID models only take account of the parameters of the PE. The discrepancy between the primary beam diameter and the diameter of the dot response suggests that the PE are not the only players involved in the deposition process, and many authors have proposed the idea that SE might be an important factor in EBID. ${ }^{6,-11}$

Our goal is to quantify and explain the role of SE in EBID lateral resolution. The problem is simplified by considering a thin target, where only the SE1 play an important role and where the SE2 can be neglected. We start the evaluation by assuming that the SEs are generated exactly in the place where the PE inelastic interaction took place, which means that the generation of SE is a perfectly localized process. We then proceed to assess the effect of the so-called delocalization of inelastic electron scattering.

In some of our simulations, we consider a zero-diameter primary beam, which is an extreme case, but ideal for the study of the ultimate spatial resolution. Fortunately, this situation can almost be achieved in practice nowadays due to the availability of modern commercial electron-optical instruments with focused beams of 0.2-nm diameter. We will try to predict the geometry of the singular dot deposited by this electron beam.

The EBID parameter that essentially dictates the shape of the singular dot is the material deposition rate onto the surface. This deposition rate $R(x)$ (in units of molecules per unit area per unit time) at any site on the surface situated at a distance $x$ from the incidence point of the primary beam is 12

$$
R(x)=\int_{0}^{E_{0}} f(x, E) \sigma_{\mathrm{diss}}(E) N d E,
$$

where $E_{0}$ is the energy of the primary electrons, $f(x, E)$ is the flux of electrons passing through the surface (electrons per unit area per sec), $\sigma_{\text {diss }}(E)$ is the electron impact dissociation cross section of the precursor, and $N$ is the molecular density on the surface. In the following sections we will describe all the steps necessary to quantify the deposition rate given by Eq. (1).

\section{ELECTRON-IMPACT-INDUCED DISSOCIATION}

As can be seen from Eq. (1), one of the key processes involved in EBID is the electron-induced dissociation of the adsorbed gas molecules. The electrons can be monoenergetic, such as the primary electrons, with energies specific to scanning electron microscopy (SEM) or transmission electron microscopy (TEM) (between 30 and $200 \mathrm{keV}$ ), or have a spectrum of energies peaked at $2-5 \mathrm{eV}$, such as the secondary electrons. In both cases, given the energy $E$ of an electron interacting with a gas molecule, an important parameter needed for the prediction of the deposited geometry is the electron-impact dissociation cross section $\sigma_{\text {diss }}(E)$. This parameter, specific for each type of precursor molecule, in practice is only known for a few gases. This is because of the difficulties in detecting the neutral products resulting 
from the dissociation, as well as the multitude of possible dissociation paths, enormously increasing with the complexity of the molecule. The most studied molecule is also the simplest one, $\mathrm{H}_{2}$, and even here there are still open questions. Furthermore, nearly complete data is available for a few simple gases, such as $\mathrm{N}_{2}$ and CO. Recently, databases have been created collecting all the available information for gases used in plasma processing in microengineering, as well as for hydrocarbons. ${ }^{13}$ Based on this information, other research groups have been able to develop scaling algorithms and general equations valid for larger groups of molecules. Nevertheless, hardly any information exists on the metalcarrying compounds used in EBID, in particular, $\mathrm{W}(\mathrm{CO})_{6}$ and $\mathrm{Fe}(\mathrm{CO})_{5}$. This lack of information caused the few authors concerned with EBID resolution to use very simplistic threshold functions to replace the real cross sections ${ }^{14,15}$ or to even abandon their research at this stage. ${ }^{12}$

We circumvent this difficulty by taking advantage of the similarity between EBID and the specimen-contamination phenomenon in electron microscopy, by replacing the unknown $\sigma_{\text {diss }}(E)$ of the metal-bearing molecules with the electron-impact dissociation cross section of a hydrocarbon molecule frequently present in the electron microscope chamber and contributing to carbon contamination. ${ }^{16} \mathrm{We}$ used a recently published ${ }^{17}$ general equation for the electronimpact dissociation cross section of $\mathrm{C}_{x} \mathrm{H}_{y}$ in the gas phase,

$\sigma_{\mathrm{diss}}(E)=\left\{\begin{array}{l}0, \quad E \leqslant E_{\mathrm{th}} \\ \sigma_{\max }\left(1-\frac{\left(E_{\max }-E\right)^{2}}{\left(E_{\max }-E_{\mathrm{th}}\right)^{2}}\right), \quad E_{\mathrm{th}}<E<E_{\max } \\ \sigma_{\max } e^{\left(E-E_{\max }\right) / \lambda}, \quad E \geqslant E_{\max } .\end{array}\right.$

This equation expresses the dissociation cross section of a hydrocarbon molecule in terms of four parameters $E_{\mathrm{th}}, E_{\max }$, $\sigma_{\max }$, and $\lambda$, making it very useful and easy to implement in a computer program.

$\sigma_{\max }$, the maximum cross section, can be calculated as a function of the number of carbon $\# \mathrm{C}$ and hydrogen $\# \mathrm{H}$ atoms present in the molecule, as follows:

$$
\sigma_{\max }=(1.89 \# \mathrm{C}+0.33 \# \mathrm{H}-0.505) \times 10^{-2}\left[\mathrm{~nm}^{2}\right] .
$$

$E_{\max }$, the energy at which the maximum cross section occurs, and $\lambda$, a constant that determines the rate of decay of the cross section beyond $E_{\max }$, are given by Alman et al. ${ }^{17}$ for many dissociation channels. For instance, for $\mathrm{C}_{2} \mathrm{H}_{5} \rightarrow \mathrm{C}_{2}$ $+\mathrm{H}_{4}+\mathrm{H}: E_{\max }=25 \mathrm{eV}$ and $\lambda=77 \mathrm{eV}$; for $\mathrm{C}_{2} \mathrm{H}_{5} \rightarrow \mathrm{C}_{2}+\mathrm{H}_{3}$ $+2 \mathrm{H}: E_{\max }=18 \mathrm{eV}$ and $\lambda=11.4 \mathrm{eV}$.

$E_{\text {th }}$ is the threshold energy for molecular dissociation and is always around $10 \mathrm{eV}$ for gas-phase hydrocarbons. When the gas molecule is adsorbed on a surface, the dissociation threshold energy is shifted to values lower than that in the gas phase. Experiments of deposition induced by low-energy electrons in a scanning tunneling microscope (STM) by Uesugi and $\mathrm{Yao}^{18}$ showed that the dissociation threshold for adsorbed hydrocarbon molecules lies at 3-4 eV. We used this information to adjust the $\mathrm{C}_{2} \mathrm{H}_{5}$ dissociation cross section

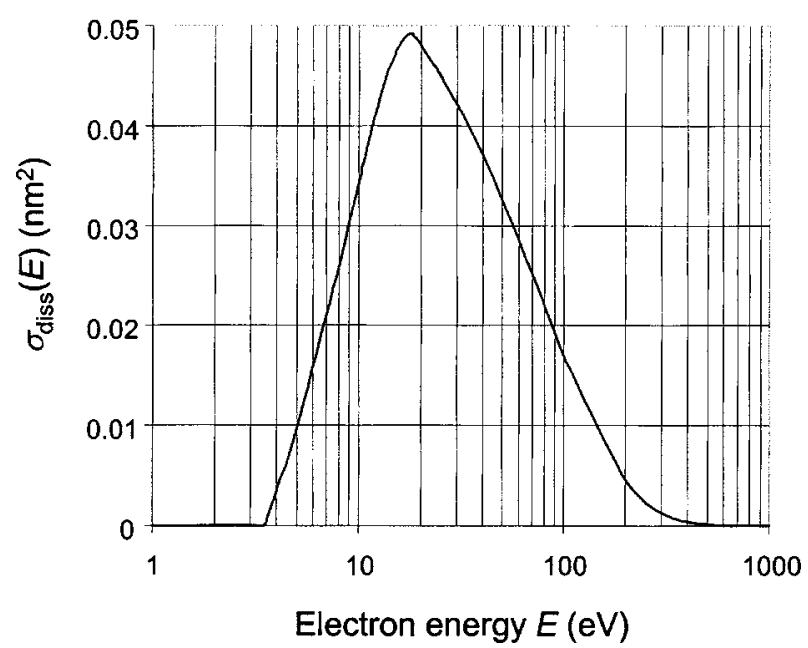

FIG. 3. The electron impact dissociation cross section $\sigma_{\text {diss }}(E)$ for adsorbed $\mathrm{C}_{2} \mathrm{H}_{5}$ as a function of the incident electron energy $E$. The general equation [Eq. (2)] was used, adapted for the adsorbed phase. [From Silvis-Cividjian (see Ref. 8).]

for the adsorbed phase. Figure 3 shows a plot of $\sigma_{\text {diss }}(E)$ for adsorbed $\mathrm{C}_{2} \mathrm{H}_{5}\left(E_{\text {th }}=3.5 \mathrm{eV}\right)$ with a $E_{\max }$ of $18 \mathrm{eV}$ and a $\lambda$ of $77 \mathrm{eV}$.

\section{THE ROLE OF THE SECONDARY ELECTRONS}

Another quantity necessary in the simulation of the deposition process is the spatial and energetic distribution of the electrons on the surface covered by gas molecules $f(x, E)$ [see Eq. (1)]. Actually, only the flux of secondary electrons needs a considerable amount of work since the flux of monoenergetic PE on the target surface can be simply approximated with a Gaussian function.

Inspired by Fig. 1, we divide the process of dot deposition, and consequently the modeling of the SE influence, into two stages: the very beginning of material deposition (A), when the secondary electrons emerge only from the top surface of the flat target, and the dot growth (A-C), when the surface from where SEs emerge starts to diverge from a flat one, taking a more complex shape. The first stage can give an indication of the ultimate EBID resolution as determined by the SE.

\section{A. Monte Carlo simulation for secondary-electron emission}

The problem of the electron-solid interaction should ideally be solved experimentally by measuring the relevant physical quantities resulting from the primary beam bombardment. However, the interaction process remains partly unknown due to the limitations of present experimental techniques. In this situation, computer simulations become the most powerful theoretical method, as an idealized replacement for an experiment. One of the most used methods for the simulation of secondary-electron emission from targets irradiated by electrons is the Monte Carlo technique. A vast literature is available on this subject, mainly for the study of image resolution in SEM or, less frequently, for the fabrication resolution in resist-based electron-beam lithography. An electron entering the target generates a complicated cascade 
multiplication of electrons. Solving the full cascade collisions tree generated in the target is a titanic work both for the computer and for the programmer. Approximate models have been generated as time savers and as a replacement for the unknown scattering phenomena.

Monte Carlo simulations consider two possible types of electron scattering events, viz., elastic and inelastic, while between two processes the electron moves linearly. Elastic scattering is assumed to only lead to an electron deviating from its trajectory with no energy loss. Elastic electron scattering can be treated using the Rutherford total cross section, ${ }^{19}$ suitable for high-energy electrons, or the Mott cross section, more suitable for low-energy electrons. ${ }^{20}$

Inelastic scattering occurs as a result of electron interaction with the electrons from the target atoms and deviates the electron, leading to energy loss, which can result in the generation of secondary electrons. Their generation can be treated using three theoretical models: (1) The direct model is the simplest one and assumes that SEs are generated only through excitations of target conduction electrons and that the SEs are generated isotropically. (2) The fast-secondaryelectron (FSE) model is intensively used and very suitable for computer implementation. It starts from the free-electron model and the Coulomb interaction between the incident electron and the free electrons in the target atoms. The FSE model assumes that SE generation is not isotropic but predominantly at relatively large angles to the beam direction. ${ }^{19}$ (3) The most complex and recently implemented model is the dielectric function model. ${ }^{21}$ This model uses the inelastic electron scattering formulated in terms of the dielectric function of the target. The dielectric function $\varepsilon(\omega, q)$ describes the dielectric response of a material to an external perturbation, such as electromagnetic waves or incident electrons. It is an attractive approach because it uses a measurable feature of the target material and thus includes almost all excitation mechanisms. A disadvantage of this model is that it can only be used for materials of known dielectric function.

The SE transport to the surface after generation can also be treated in different ways. The straight-line approximation (SLA) is a very simple transport model proposed by Chung and Everhart. ${ }^{22}$ It is based on the assumption that SEs propagate linearly towards the surface and treats each scattering event as an electron absorption. This assumption is realistic for low-energy SE. The absorption effect is simulated with an exponential decay, yielding the probability that an electron at depth $z$ and energy $E$ will reach the surface with the same energy. This model can be improved by taking into account the cascades produced in the target. The model proposed by Koshikawa and Shimizu ${ }^{23}$ is still intensively used.

\section{B. The role of secondary electrons from the flat target surface}

We have written a Monte Carlo simulation program using the DELPHI for Windows programming environment. The model we used is the fast-secondary-electron model with cascades included. It has been chosen as a compromise between precision and computing time. The simulated trajectories of the secondary electrons in the target are plotted on- (a)

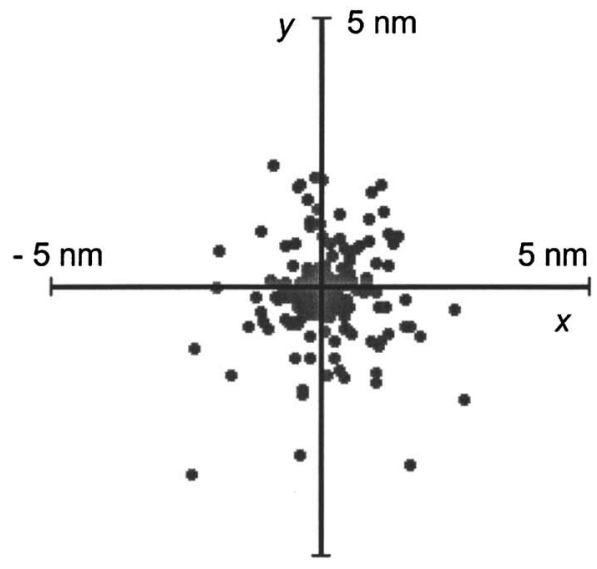

(b)

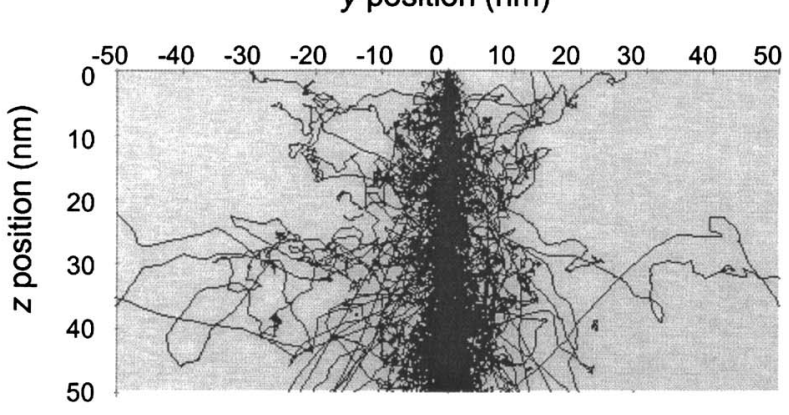

FIG. 4. Graphical results of our Monte Carlo simulations. (a) The exit sites of SE on the surface of a 100-nm target irradiated by a zero-dimensional $20-\mathrm{keV}$ beam. (b) 5000 trajectories of secondary electrons for a zerodimensional $100-\mathrm{keV}$ primary beam and a $50-\mathrm{nm} \mathrm{Cu}$ target ( $Y-Z$ plane).

line, together with their exit sites on the target surface (see some examples in Fig. 4). One of the numerical results of our Monte Carlo simulations is the spatial distribution of SE on the top flat target surface, an example of which is shown in Fig. 5 for a zero-dimensional electron beam of $20 \mathrm{keV}$, irradiating a $10-\mathrm{nm}$-thick $\mathrm{Cu}$ target. The spatial distribution is graphically presented in three ways. $N(r)$ is the total SE current emerged on the surface in a ring of radius $(r, r+d r) . I(r)$ is the radial current density of SE. $N_{\text {tot }}(r)$ is the integral function of $N(r)$, showing the number of SE emerged from a circle of radius $r$.

It can be seen in Fig. 5 that the SE radial distribution peaks around the origin, indicating that the SEs emerge clustered very close to the point of the primary beam incidence. For this reason we think that the spatial distribution of SE can be more realistically described by the full width $\mathrm{FW}_{50}$ of the distribution, or by the diameter where $N_{\text {tot }}(r)=0.5$, equal to the diameter of the disk from where $50 \%$ of the SEs are emitted. Figure 5 shows that even in the case of a zerodimensional primary beam, $50 \%$ of the SEs emerge from a disk of almost 3-nm diameter.

This result is similar to the results obtained previously by Joy ${ }^{19}$ and Ding and Shimizu, ${ }^{24}$ who also explored SE emission from irradiated targets. This only indicates that our Monte Carlo simulations are correct but does not yet lead to a conclusion regarding EBID resolution. In order to be useful 


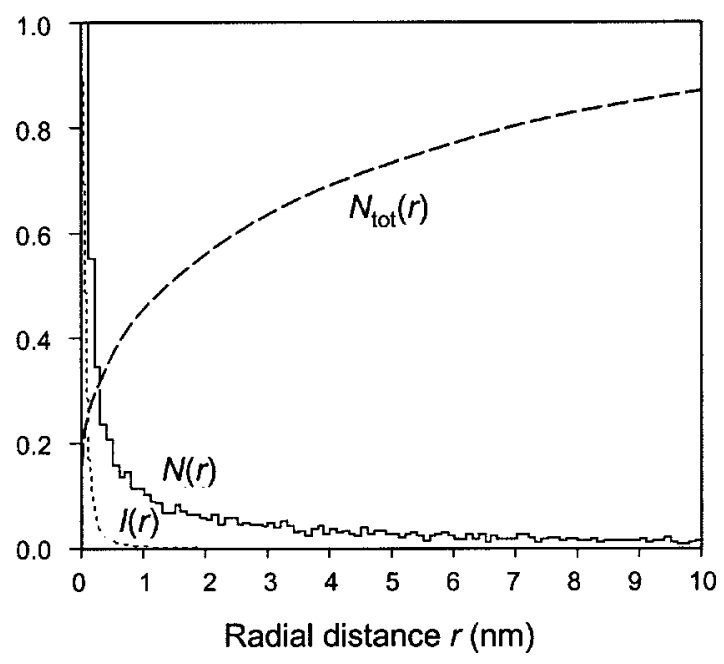

FIG. 5. Results from Monte Carlo simulations of secondary-electron emission, showing the radial spatial distribution of secondary electrons on the top surface of a 10 -nm-thick $\mathrm{Cu}$ target irradiated by a zero-dimensional electron beam accelerated at $20 \mathrm{keV} . N(r)=$ the total SE current, $I(r)=$ the radial surface density distribution, and $N_{\text {tot }}(r)=$ the total number of SE emerged from a circle of radius $r$. [From Silvis-Cividjian (see Ref. 8).]

in EBID resolution analysis, the Monte Carlo simulation also has to provide the energy-space distribution $f(x, E)$ of the secondary electrons. This kind of requirement is not typical for the majority of SEM simulations.

The procedure continues by combining the SE spatial and energetic information obtained from Monte Carlo simulations $f(x, E)$ with the electron-impact dissociation cross section of the precursor $\sigma_{\text {diss }}(E)$ as in Eq. (1). Only in this way can the profile of the dot deposited in the very beginning of the process be obtained.

By convolving this result with the Gaussian distribution of the PE on the target surface, we can obtain the profile of a dot deposited by the secondary electrons for any Gaussian beam of a given diameter.

Figure 6 shows two examples of dot cross-sectional profiles, one generated by a 2-nm-diam beam, an exponent of a typical modern SEM performance often reported by EBID users, and one by a $0.2-\mathrm{nm}$ beam, representing the state-ofthe-art in a scanning transmission electron microscope (STEM), never used for EBID yet.

As best measure of the EBID resolution we consider the area on the target containing $50 \%$ of the dissociated molecules. From Fig. 6 we see that the role of SE in the enlargement of the dot diameter is negligible when the primary beam is around $2 \mathrm{~nm}$ (the dot also has a $2-\mathrm{nm} \mathrm{FW}_{50}$ ), but becomes significant for sharper beams, around $0.2 \mathrm{~nm}$, causing an enlargement of nearly $25 \%\left(\mathrm{FW}_{50}=0.23 \mathrm{~nm}\right)$. These are almost the same results as we obtain when higher generations of electrons, generated by cascades, are taken into account. By comparing to Fig. 5, we observe that the spatial distribution of the SE is not identical to the spatial distribution of the dissociated molecules on the surface forming a dot. This is why it is important to combine the spatial information for SEs with their power to dissociate precursor molecules, especially for very sharp, quasi-one-dimensional beams. (a)

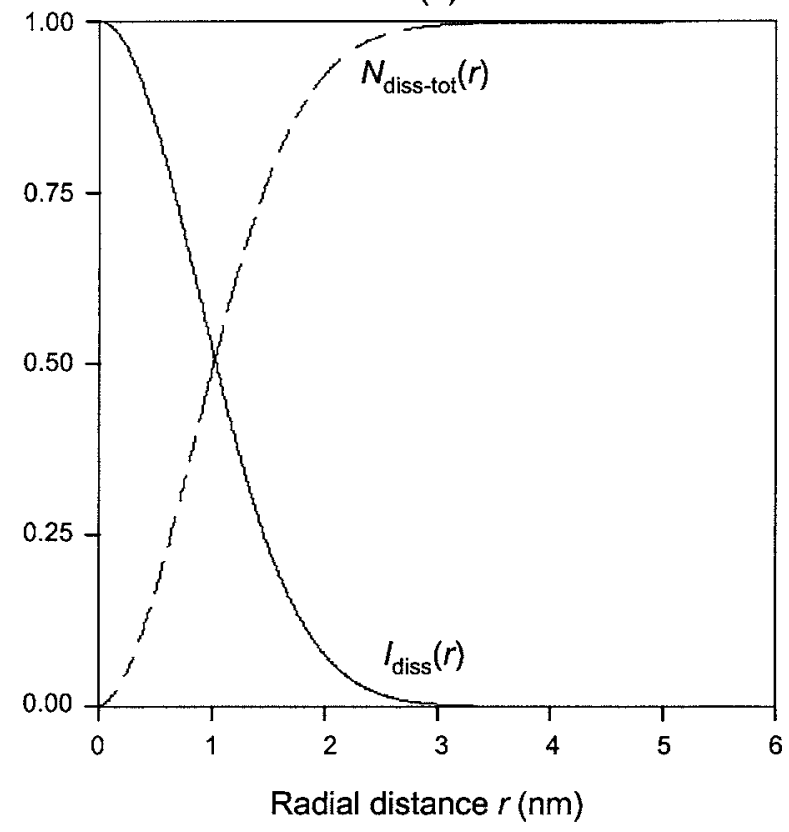

(b)

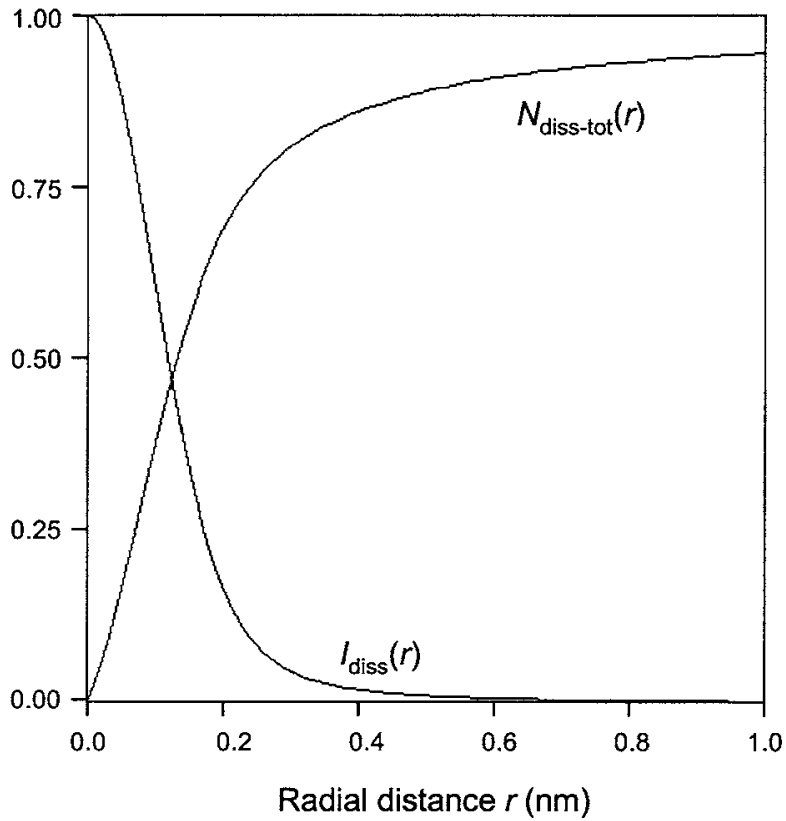

FIG. 6. The normalized profile of a singular dot fabricated using EBID by a 20-keV electron beam with (a) 2-nm diameter and (b) 0.2-nm diameter on a 10 -nm-flat $\mathrm{Cu}$ target. Here $I_{\text {diss }}(r)$ is the normalized radial density distribution of the dissociated molecules on the surface and $N_{\text {diss-tot }}(r)$ is the normalized radial integral function, showing the number of dissociated molecules on a disk of radius $r$. The very beginning of the growth process is analyzed. [From Silvis-Cividjian (see Ref. 8).]

The lateral size of the dot at the initial stage A (see Fig. 1) indicates the ultimate EBID resolution obtainable. Taking into account that an atom has a diameter of approximately $0.3 \mathrm{~nm}$, the ultimate EBID resolution may be of atomic dimension.

Hübner et al. ${ }^{6}$ and Hiroshima and Komuro ${ }^{25}$ reported EBID experiments with a $2-\mathrm{nm}$ beam producing dots with a minimum lateral size of $15-20 \mathrm{~nm}$. Our simulation results indicate that only the SE emerged from the target surface 


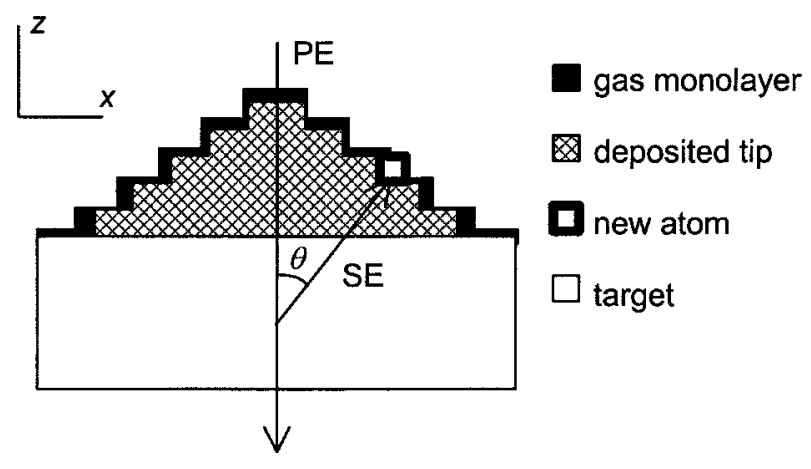

FIG. 7. Geometry used by the cellular automata profile simulator. A primary electron enters the object (target+tip). As a result, secondary electrons (SEs) are emitted from the object. In the place where they exit the object, an adsorbed molecule can be dissociated and a new atom is deposited. Consequently, the border of the object is adjusted and the process continues with a new primary electron.

cannot explain the difference between the beam diameter and the diameter of the deposited dot. We believe that this difference arises from the fact that the SEs emerge from a surface progressively diverging from a flat one. We also believe that the reported values were not measured in the very beginning of the deposition (moment A, Fig. 1) but at a moment randomly situated on the segment A-C. This is why we recommend always reporting EBID lateral resolution results as a pair of values (diameter, time) or (diameter, height).

\section{The role of the secondary electrons scattered in the tip}

We will try to demonstrate that the SE that emerged from the lateral flanks of the grown tip are responsible for the shape shown in Fig. 1. The evaluation of the role of SE in EBID resolution continues with a simulation of the temporal evolution of the dot geometry, according to the following scenario. While a tip is growing, secondary electrons can emerge from its lateral flanks also, dissociating the molecules adsorbed on their exit sites and depositing an atom at that location (see Fig. 7). Thus the tip will grow not only vertically, but also laterally. Saturation of the diameter growth will occur when the SEs do not have enough energy to exit the lateral flanks. The electron mean free path in the target material $^{26}$ gives this saturation value.

A profile simulator can verify this scenario. The methods used to simulate the profile evolution during deposition or etching processes in microelectronics can be divided into two categories, viz., cellular automata (CA) and geometric methods. Geometric methods treat the substrate as a continuous entity. Examples of geometric methods are the string and the set level algorithms. ${ }^{27,28}$

Cellular automata are computational tools mainly used in biology to simulate the evolution of living cells, but they can also be applied to several physical problems where local interactions are involved. ${ }^{29,30} \mathrm{CA}$ are idealizations of physical systems, in which space and time are discrete and interactions are local. A CA consists of a regular uniform $n$-dimensional lattice (or array). Each cell is restricted to a local neighborhood only, being incapable of immediate global communication. We have developed a simulation pro- gram using a two-dimensional CA for the spatial evolution of a dot grown by EBID in the spot mode of an electron microscope. The idea of using a profile simulator in the study of EBID resolution is rather new. Only one group has applied the string algorithm to EBID in order to simulate the crosssectional profile of a line deposited by a scanned focused beam. ${ }^{25}$ We consider our approach to be more detailed because we trace the SEs from their internal generation until their exit on the surface, instead of using an analytical expression for their yield at the surface.

The profile simulator has been written using the DELPHI programming language and runs as a Windows application. As an example, we consider the growth of a carbon contamination dot by a zero-diameter beam on a thin carbon foil covered with $\mathrm{C}_{2} \mathrm{H}_{5}$ precursor. The same material is chosen both for the substrate and the deposit in order to avoid complicated interface effects. This situation is possible to experience in practice, at high acceleration voltage in a modern high-resolution STEM, which enables checking the simulation against experiments. The geometry used by the profile simulator is presented in Fig. 7. The substrate together with the already deposited tip form one object permanently covered by a border of one monolayer of gas molecules. This is an ideal situation when the diffusion time necessary for a gas molecule to reach the irradiated area from the sink is assumed to be zero. In order to save time, we used the simplest theoretical models for the SE analysis, viz., the direct model for the SE generation and the SLA model for their transport to the surface.

A sequence of cross-sectional profiles of the dot simulated at regular intervals of time is presented in Fig. 8(a). Based on these plots, Fig. 8(b) shows the measured dot diameter at base and at half maximum and the dot height. Obviously, the diameter indeed grows steeply until it reaches a saturation value of 20-30 $\mathrm{nm}$. We conclude that in EBID the SEs play a decisive role in shaping a singular dot and thus impose a fundamental limit to EBID resolution.

\section{THE ROLE OF DELOCALIZATION OF ELECTRON INELASTIC SCATTERING}

In the previous section, we estimated the EBID resolution determined by the secondary electrons generated during the irradiation process. We recall that at that moment we assumed that the SEs were generated at the same place where the primary inelastic electron interaction took place. In reality, inelastic electron scattering in matter is a delocalized process, which is inversely proportional to the electron energy loss. As a result, all processes initiated by this energy loss, especially when the energy loss is small, like in the generation of secondary electrons or the degradation of gas molecules, are also delocalized. Difficulties in the theoretical modeling of delocalization are imposed by the unknown elements in inelastic electron scattering in a solid or gas. Often, very simple models are used to quantify the delocalization effects, mainly for studies of SEM or STEM imaging resolution. The results of different approaches sometimes differ drastically, leading to pessimistic as well as optimistic predictions. For example, the theoretical estimates for the delocalization of secondary-electron generation range from 


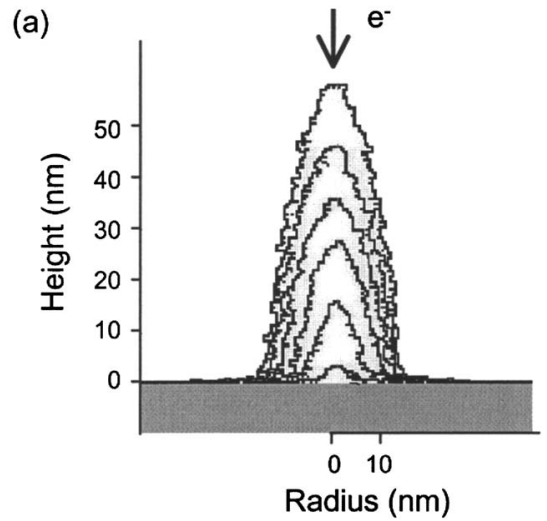

(b)

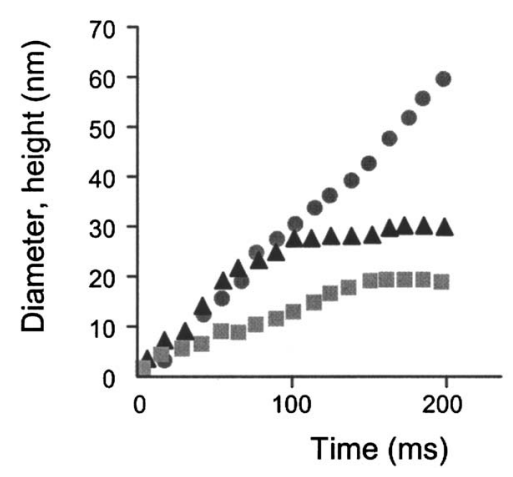

FIG. 8. A sequence of simulated profiles for a singular contamination dot grown by a zero-diameter electron beam, accelerated at $200 \mathrm{keV}$ and normal incident on a $10-\mathrm{nm}$ thick carbon foil; the time intervals corresponding to each profile are in the sequence of 18-54-90-126-162-198 ms. (a) Sequence of cross-sectional profiles. (b) Time evolution of the dot geometry: the dot height (circles), the dot diameter measured at the base (triangles), and the dot diameter measured at half its maximum height (squares). Saturation in the diameter is clearly observed.

0.05 to $70 \mathrm{~nm}$, but in practice is somewhere in between these two extremes. ${ }^{31}$ The predicted range approaches the order of magnitude of the smallest electron-beam diameter obtainable nowadays in a STEM, around $0.2 \mathrm{~nm}$. That is why it is feared that the delocalization of inelastic electron scattering imposes a fundamental limit on any analysis technique performed in any electron microscope, as well as any electronbeam-induced process in general, and EBID in particular. One of the few attempts to study the localization of energy transfer between electrons and polymethylmethacrylate (PMMA) resist in electron-beam lithography was reported by Han and Cerrina. ${ }^{32}$

In this section, we describe our attempt to quantify the role of SE delocalization in EBID resolution. During the past years, several approaches have been formulated for the evaluation of the degree of electron-energy-transfer delocalization. Their complexity increases from an almost straightforward one, the classical model, via a semiclassical model, to a very complicated quantum-mechanical approach. We will investigate which model gives satisfactory results for the problem of delocalization in EBID. According to Ritchie and Howie, ${ }^{33}$ electrons with energies between 10 and $100 \mathrm{keV}$ behave as classical point-charge particles and quantum corrections are negligible. Therefore, we will only describe the first two models.

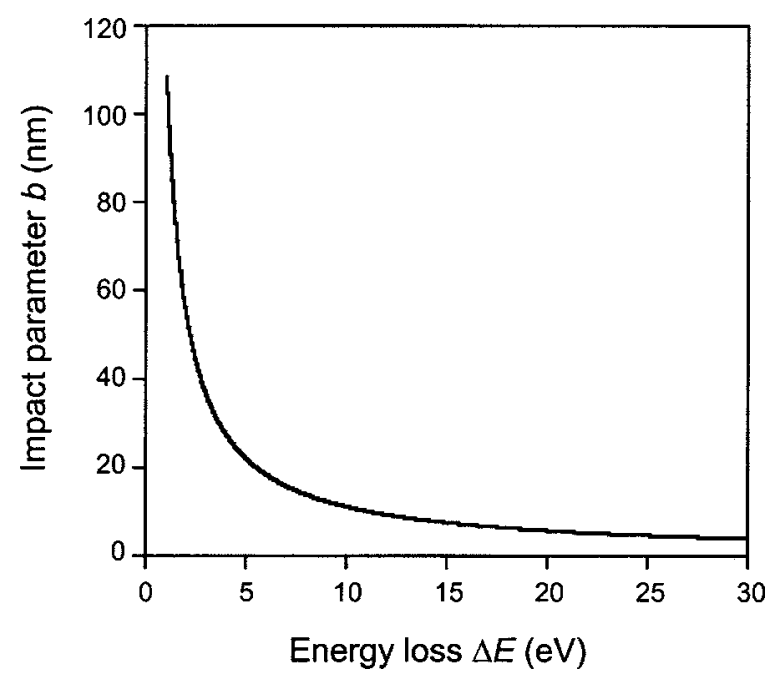

FIG. 9. Impact parameter $b_{\max }(\Delta E)$ for a $100-\mathrm{keV}$ electron according to the classical model.

\section{A. The classical model}

The most frequently used model is the classical model, which treats the electron as a point charge with energy $E$, passing next to an atom $A$ and losing some energy $\Delta E$. The impact parameter $b$, known also as the Bohr cut-off radius, is defined as the radius of a sphere inside which the electron must pass for the interaction to really happen. The magnitude of the impact parameter can be straightforwardly estimated from the wave nature of the electron and the Heisenberg uncertainty principle. The maximum impact parameter is given by Colliex, ${ }^{34}$

$$
b_{\max }=\frac{\hbar v}{\Delta E},
$$

where $\hbar$ is Planck's constant, $v$ is the velocity of the incident electron, and $\Delta E$ is the energy lost by the incident electron.

Figure 9 shows the dependence of the impact parameter $b_{\max }$ on the electron energy loss $\Delta E$ for the case of a 100 $-\mathrm{keV}$ electron. From Eq. (4) we can estimate, for example, that an electron having a velocity of $v=0.5 c$ can lose an energy of $\Delta E=5 \mathrm{eV}$ within an impact parameter $b_{\max }$ of ca. $20 \mathrm{~nm}$ and can lose an energy of $\Delta E=25 \mathrm{eV}$ within an impact parameter $b_{\max }$ of ca. $4 \mathrm{~nm}$. By applying this simple model to EBID, we might conclude that a dissociation process, which needs an energy loss of $5 \mathrm{eV}$, could give EBID features limited in resolution by the delocalization to $20 \mathrm{~nm}$. It is generally accepted now that the classical model overestimates the delocalization.

\section{B. The semiclassical model}

The semiclassical model treats the electron as a point charge with a well-defined straight linear trajectory and constant velocity. The interaction between the electron and the atom is treated quantum mechanically. The classical relation between the impact parameter $b$ and the energy loss $\Delta E(b)$ is not correct anymore because in quantum mechanics only a certain probability of interaction makes sense. A new quan- 

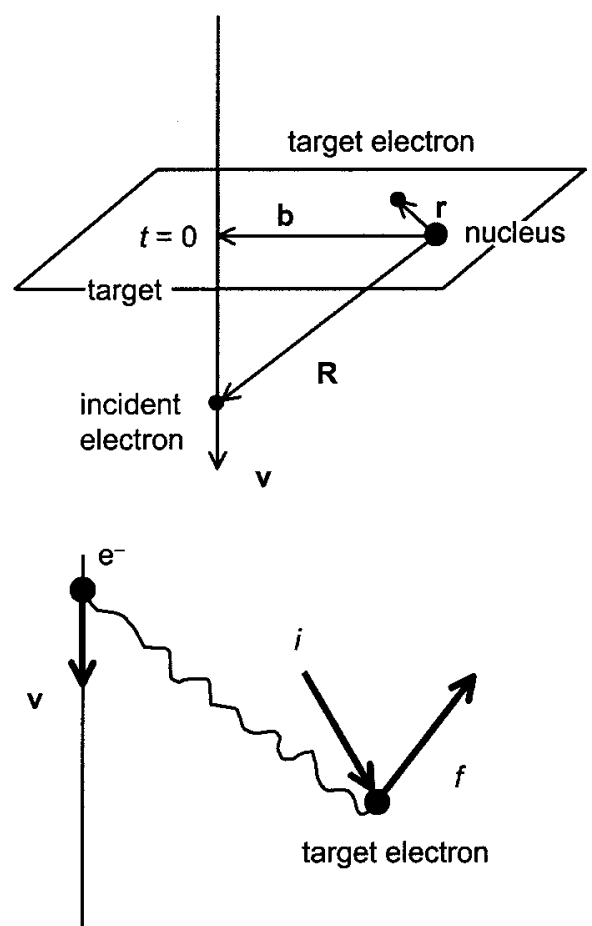

FIG. 10. Feynman diagram of electron-medium interaction. A fast electron passes with a velocity $v$ near a target atom, which, as result of energy transfer, jumps from its initial state $i$ to final state $f$. [From Silvis-Cividjian (see Ref. 8).]

tity is thus introduced, $P(b, E)$, given by the probability for a swift electron to lose a discrete energy $E$ in an interaction with a target atom situated at a distance $b$.

The origin of coordinates is considered to be in the center of mass of the target atom, its nucleus (see Fig. 10). The nearest approach at the impact parameter $b$ is at time $t=0$. For simplicity, a hydrogenlike atom is considered, with only one electron. The impact vector $\mathbf{b}$ and velocity vector $\mathbf{v}$ are mutually perpendicular vectors. The trajectory of the incident electron can be expressed as

$$
\mathbf{R}=\mathbf{b}+\mathbf{v} \cdot t,
$$

where $\mathbf{R}$ is the coordinate vector of the incident electron.

The Hamiltonian characterizing the interaction between the fast electron and the target is given by

$$
V(t)=\frac{e^{2}}{|\mathbf{b}+\mathbf{v} t-\mathbf{r}|},
$$

where $\mathbf{r}$ is the coordinate vector of the electron in the excited atom.

The atom is considered in the ground state until time $t$ $=0$, when the swift electron field is suddenly "switched on." During the interaction, the incident electron loses an energy $E$ by transferring it to the target atom situated at distance $b$. The result of this energy transfer is that the atom makes a transition from the initial ground state $(i)$ to a final higherenergy state $(f)$ (see Fig. 10), described by wave functions $\varphi_{i}$ and $\varphi_{f}$, respectively. The calculation of these wave functions is a very difficult and time consuming, sometimes even impossible operation. For this reason usually only hydrogenlike atom wave functions are used.
Time-dependent perturbation theory gives the transition probability that an atom experiences a transition $i \rightarrow f$ under the influence of a Coulomb field of a classical point electron, traveling with a constant velocity $v$ along a path specified by the impact parameter $b,^{33}$

$$
P_{i \rightarrow f}=\frac{1}{i \hbar^{2}}\left|\int_{-\infty}^{\infty}\left\langle\varphi_{i}|V(t)| \varphi_{f}\right\rangle e^{\left[i\left(E_{f}-E_{i}\right) t\right] / \hbar}\right|^{2},
$$

where the energy transfer necessary for the transition is $E$ $=\hbar \omega=E_{f}-E_{i}$.

The dimensions of atoms and molecules are small. Because the origin of $\mathbf{r}$ is in the center of the atom, the exponential $e^{i \omega t}=e^{i \mathbf{k r}}$ can be expanded as a power series, since this series will converge very rapidly:

$$
e^{i \mathbf{k r}}=1+\mathbf{k r}-i(\mathbf{k r})^{2}+\ldots .
$$

The classical analog of this expansion of the exponential is a multiple expansion of the charges that interact with the radiation field. The transitions that are due to the first term of the expansion are called electric dipole transitions and the transitions due to the second term are known as magnetic dipole and electric quadrupole transitions. ${ }^{35}$ The so-called dipolar approximation, which only takes into account the electric dipole transitions, is very widely used. The dipolemoment operator is defined as er. The matrix element of the dipole-moment operator between the target states $i$ and $f$, $r_{f i}=\left\langle\varphi_{f}|\mathbf{e r}| \varphi_{i}\right\rangle$, is responsible for the electric dipole transitions between the two states and known as the transition moment. $^{36}$

Time-dependent perturbation theory in the dipolar approximation gives the probability that the atom electron will be excited from the ground state $i$ to state $f::^{37}$

$$
\begin{aligned}
P_{(i \rightarrow f)}= & \frac{1}{\pi^{2} b_{\max }^{2}}\left(\frac{\lambda_{0}}{a_{0}}\right)^{2}\left[\left(\mathbf{r}_{f i} \cdot \mathbf{e}_{v}\right)^{2} K_{0}^{2}\left(b / b_{\max }\right)\right. \\
& \left.+\left(\mathbf{r}_{f i} \cdot \mathbf{e}_{b}\right)^{2} K_{1}^{2}\left(b / b_{\max }\right)\right],
\end{aligned}
$$

where $\lambda_{0}=h / m v$ is the nonrelativistic de Broglie wavelength of the incoming electron, $a_{0}$ is the Bohr radius, $\mathbf{e}_{v}$ and $\mathbf{e}_{b}$ are the unit vectors of the $\mathbf{v}$ and $\mathbf{b}$ directions, $\mathbf{r}_{f i}=\left\langle\varphi_{f}|\mathbf{r}| \varphi_{i}\right\rangle$ is the dipole matrix element of target electron coordinate operator $\mathbf{r}, K_{0}(x)$ and $K_{1}(x)$ are the modified Bessel functions of the second kind, and $b_{\max }=v \hbar / E_{f i}$ is the Bohr cut-off parameter.

The unknown quantities in this expression are the dipole matrix elements $\mathbf{r}_{f i}$, which are difficult to calculate. Using the approximation suggested by Fano ${ }^{38}$ and Hameka ${ }^{35}$ we can assume that the atoms in the target are randomly oriented. In this approximation the components of the dipole matrix elements are equal to one another and can be expressed in terms of the oscillator strength $f_{f i}$, according to the relation

$$
\left(r_{f i}\right)_{v}^{2}=\left(r_{f i}\right)_{b}^{2}=\frac{\hbar^{2}}{2 \pi E_{f i}} f_{f i},
$$

where $f_{f i}$ is the oscillator strength for the transition between initial and final states $i \rightarrow f$, a property of the target material that quantifies its response to an electromagnetic perturbation. 
Replacing these dipole matrix elements in Eq. (9), we obtain the transition probability,

$$
\begin{aligned}
P\left(b, E_{f i}\right)_{(i \rightarrow f)}= & \frac{1}{\pi^{2} b_{\max }^{2}}\left(\frac{\lambda_{0}}{a_{0}}\right)^{2} \frac{\hbar^{2}}{2 m E_{f i}} f_{f i}\left[K_{0}^{2}\left(b / b_{\max }\right)\right. \\
& \left.+K_{1}^{2}\left(b / b_{\max }\right)\right] .
\end{aligned}
$$

In order to plot the transition probability $P(b, E)$, we can simplify its expression by taking $f_{f i}=1$, an assumption valid for a bound electron that oscillates harmonically in three dimensions. In STEM calculations the oscillator strength is often modeled simply as unity up to a cutoff angle and as zero for larger collection angles. ${ }^{39}$

The indetermination that occurs in the origin of the $P(b, E)$ plot can be eliminated if $b$ is replaced with $\sqrt{b_{\min }^{2}+b^{2}}$, where $b_{\min }=1 / q \theta_{c}(E), q=\sqrt{2 m E_{P}} / \hbar$, and $\theta_{c}$ $=\sqrt{E / E_{P}}$, where $E_{P}$ is the kinetic energy of the incident electron. This idea can be found in "Classical Electrodynamics" by Jackson. ${ }^{40}$ After this correction, Fig. 11 is obtained, showing two dependencies $P(b, E)$ for two different energy losses: $E=25 \mathrm{eV}$ and $E=100 \mathrm{eV}$. The impact parameter is much smaller than in the classical approach for the same energy loss.

\section{The role of the delocalization of secondary- electron generation}

In principle, delocalization can influence the EBID resolution in many ways. For example, a PE can excite a precursor molecule situated at a distance $b$ away from its trajectory. This is the delocalization of electron-induced dissociation. The electron-induced target excitation can also be delocalized. A secondary electron can be excited in an atom situated at a distance $b$ from the PE trajectory. As a result, the spatial distribution of the SE calculated by our Monte Carlo simulations becomes wider and causes an enlargement of the deposited singular dot. Because we discovered that secondary electrons play a crucial role in EBID resolution, we should try to roughly estimate how large this enlargement might be. The problem is reduced to the estimation of the probability that a SE will be generated by exciting a target atom situated at a distance $b$ from the PE inelastic scattering center. We denote this probability as $P_{\mathrm{SE}}(b, E)$. As a case study, we consider EBID performed in a STEM, with a finely focused electron beam, accelerated at $100 \mathrm{keV}$, and passing through a thin carbon film. In order to solve this problem we use the semiclassical model. Muller and Silcox ${ }^{41}$ used this model to calculate the localization of the electron-energy-loss spectroscopy (EELS) signal and obtained results agreeing with experiments. Together with the observation of Ritchie and Howie $^{33}$ that a $100-\mathrm{keV}$ electron behaves as a classical particle, we have enough reasons to believe that the semiclassical approach is the right choice to solve the problem of SE delocalization in EBID.

Starting by replacing $b_{\max }$ in Eq. (11) from the semiclassical model, we obtain the probability that an atom will transit from an initial state 0 to a final state $n$ as a result of the incident PE passing at distance $b$, (a)

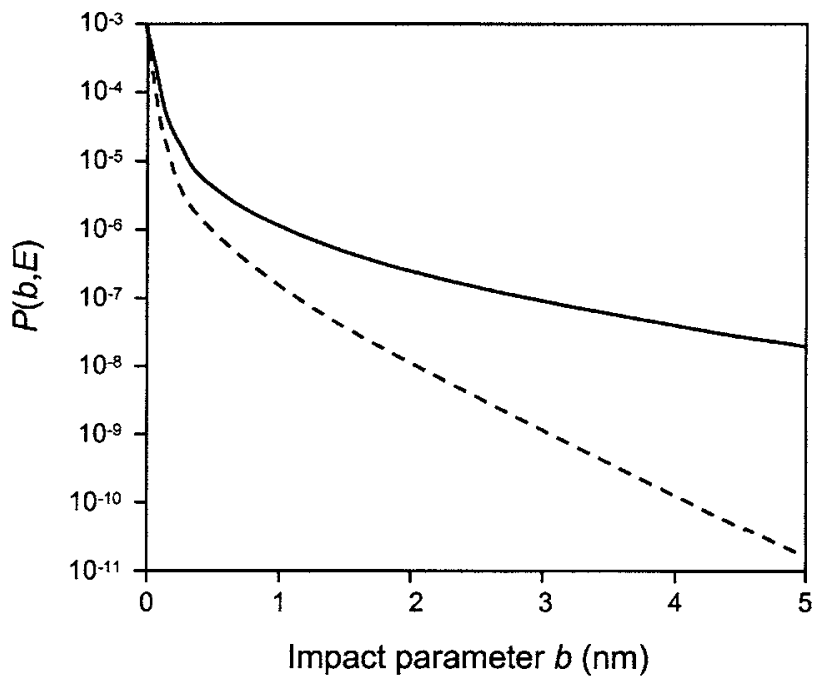

(b)

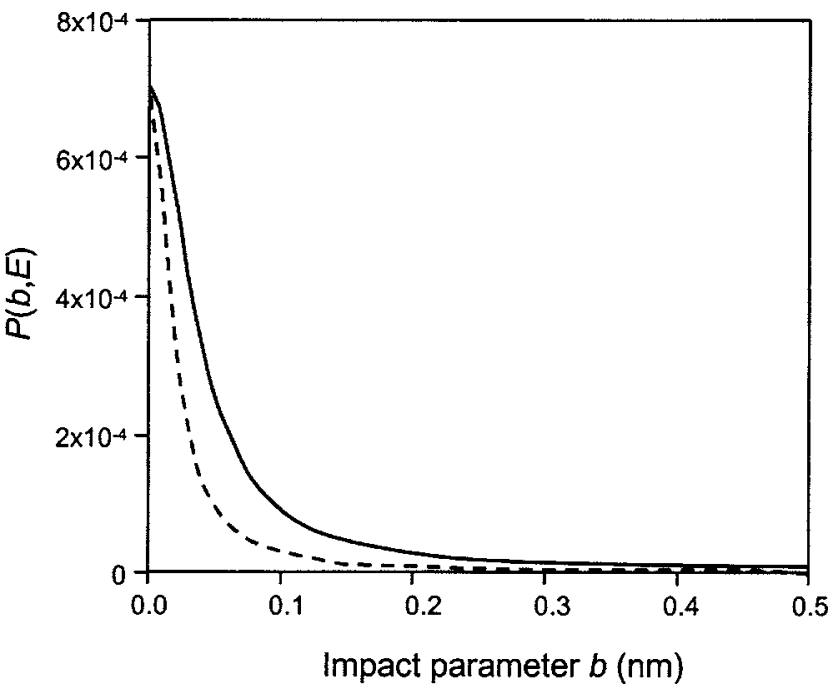

FIG. 11. The probability $P(b, E)$ that an electron with energy $E_{P}$ $=100 \mathrm{keV}$ will lose an energy of $E=25 \mathrm{eV}$ (solid lines) and $E=100 \mathrm{eV}$ (dashed lines) at an impact parameter $b$ (semiclassical approach, where the oscillator strength is assumed equal to unity). Two regions are shown separately: (a) $0-5 \mathrm{~nm}$ and (b) $0-0.5 \mathrm{~nm}$. [From Silvis-Cividjian (see Ref. 8).]

$$
\begin{aligned}
P_{(0 \rightarrow n)}= & \frac{E_{n 0}}{\pi^{2} v^{2}}\left(\frac{\lambda_{0}}{a_{0}}\right)^{2} \frac{1}{2 m} f_{n 0}\left[K_{0}^{2}\left(b E_{n 0} / \hbar v\right)\right. \\
& \left.+K_{1}^{2}\left(b E_{n 0} / \hbar v\right)\right] .
\end{aligned}
$$

If we assume that the excitation of the target atom from state 0 to state $n$ will generate a SE with energy $E=E_{n 0}$, then the probability that a SE is generated, causing an electron energy loss $E$ at distance $b$, is

$$
\begin{aligned}
P_{\mathrm{SE}}(b, E)= & \frac{E}{\pi^{2} v^{2}}\left(\frac{\lambda_{0}}{a_{0}}\right)^{2} \frac{1}{2 m}\left[K_{0}^{2}(b E / \hbar v)\right. \\
& \left.+K_{1}^{2}(b E / \hbar v)\right] f_{\mathrm{SE}}(E) .
\end{aligned}
$$

From Eq. (13) we observe that the probability $P_{\mathrm{SE}}(b, E)$ is the product of a kinematic component dependent on the incident electron motion, which can be easily evaluated, and a component dependent on the target material, $f_{\mathrm{SE}}(E)$. This is 


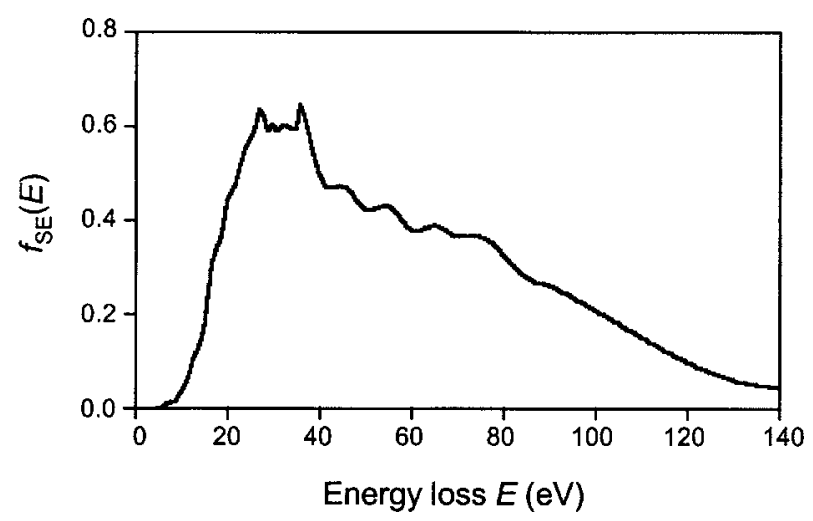

FIG. 12. The probability that a SE is generated as result of an energy loss $E$ of the primary electron. $f_{\mathrm{SE}}(E)$ is obtained as a fit parameter between a theoretical and experimental curve. [From Silvis-Cividjian (see Ref. 8).]

an almost unknown quantity, a kind of oscillator strength for the SE generation or the target probability of absorbing some energy in order to produce a SE. Our next goal is to estimate this quantity as accurately as possible.

We can calculate the integrated probability that a SE will be generated and cause an energy loss $E$ for the incident PE, $\sigma_{\mathrm{SE}}(E)$, by integrating Eq. (13) over all possible impact parameter $b$ values, considering that on our model only one atom is excited at each interaction:

$$
\sigma_{\mathrm{SE}}(E)=2 \pi N \int_{0}^{\infty} P_{\mathrm{SE}}(b, E) b d b,
$$

where $N$ is the atomic density (atoms/unit area).

First we assume that $f_{\mathrm{SE}}(E)=1$, as in many microscopy calculations, and use Eq. (14) to calculate an approximate probability that a SE will be generated with an electron energy loss $E$. The idea is to compare this dependence with a measured one in order to find the real $f_{\mathrm{SE}}(E)$. EELS is the current technique to measure the total energy lost by a primary electron passing through a target. In order to clarify the internal mechanisms of the generation of secondary electrons, a useful experiment should measure the correlation between the SE emission and the incident electron-energyloss events. The result of such an experiment is the probability that a SE will be generated by causing a certain energy loss for the primary electron. Fortunately, a few such experiments have been done and we can use data given by Drucker et $a l^{42}$ and Pijper and Kruit. ${ }^{43}$ By comparing the approximate dependence with the one measured at the same conditions, we obtain $f_{\mathrm{SE}}(E)$ as a correction factor necessary to fit both curves. Figure 12 shows the correction factor $f_{\mathrm{SE}}(E)$ as a function of the primary electron energy loss $E$.

The corrected $f_{\mathrm{SE}}(E)$ can now be introduced in Eq. (12) and we can obtain the SE delocalization profile by integrating the generation probability $P_{\mathrm{SE}}(b, E)$ over all possible energy losses:

$$
P_{\mathrm{SE}}(b)=\int_{0}^{\infty} P_{\mathrm{SE}}(b, E) d E .
$$

The normalized probability $P_{\mathrm{SE}}(b)$ that a $\mathrm{SE}$ is generated within an impact parameter $b$ from the PE inelastic scattering center is plotted in Fig. 13. We see from Fig. 13 that this (a)

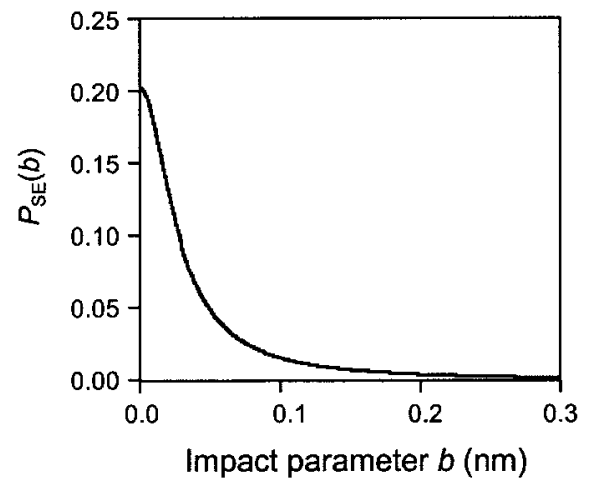

(b)

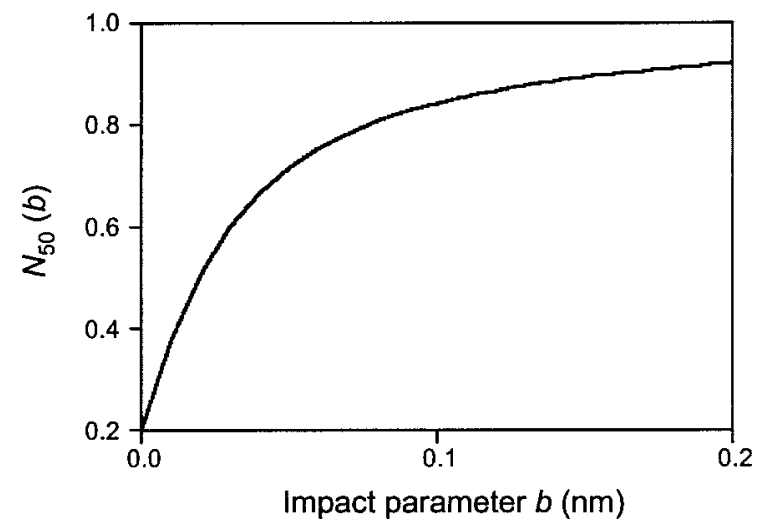

FIG. 13. (a) The normalized probability that a SE will be generated within an impact parameter $b$ (100-keV electron incident on 10-nm carbon foil); (b) the integral function of this probability: the probability that a SE will be generated within a circle of radius $b$. [From Silvis-Cividjian (see Ref. 8).]

probability decreases very sharply in the region of $0-0.05 \mathrm{~nm}$. From the plot of the integral function $N_{50}(b)$, we estimate the $\mathrm{FW}_{50}$ of the $\mathrm{SE}$ delocalization profile to be $0.04 \mathrm{~nm}$ and its $\mathrm{FW}_{90}$ to be $0.32 \mathrm{~nm}$.

In conclusion, we estimate that the delocalization of secondary-electron generation cannot enlarge the $\mathrm{FW}_{50}$ of the spatial profile with more than $0.05 \mathrm{~nm}$ and does not impose a fundamental limit to EBID resolution.

The delocalization of the secondary-electron generation was treated as a single excitation event. A complete analysis should also include the influence of the delocalization of surface plasmon generation, which is a collective excitation event. This problem is very complex and still insufficiently elucidated. Perhaps some of this effect has been taken into account by using $f_{\mathrm{SE}}(E)$ from an experiment that obviously included plasmon excitation as an intermediate process in $\mathrm{SE}$ generation. We looked for independent estimates of plasmon delocalization and studied two separated cases: (1) a 100 $-\mathrm{keV}$ primary electron excites a surface plasmon on the target situated in vacuum at distance $b$ and (2) a $100-\mathrm{keV}$ primary electron excites a surface plasmon on a precursor molecule situated in vacuum at a distance $b$ and dissociates it. We used very simple models available for these two situations, namely, those published by Aristov et al. ${ }^{44}$ Howie, ${ }^{45}$ and Ferrell and Echenique. ${ }^{46}$ We calculated the probability that the primary electron will excite a surface plasmon on the target and on the precursor molecule, respectively, at an im- 


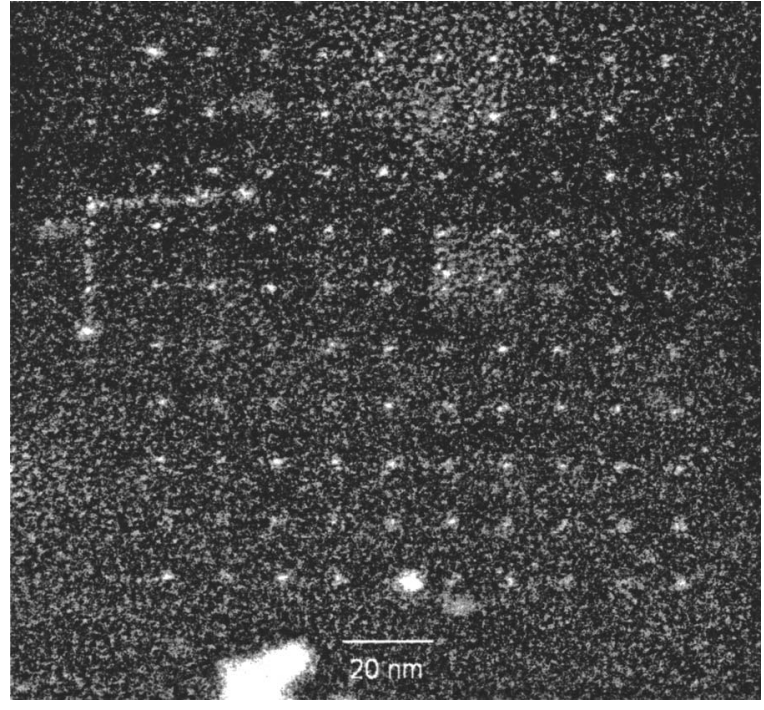

FIG. 14. HAADF image of $10 \times 10 \mathrm{~W}$ dots deposited on $30-\mathrm{nm} \mathrm{Si}_{3} \mathrm{Ni}_{4}$.

pact parameter $b$. The results show that this probability distribution's $\mathrm{FW}_{50}$ is around $1 \mathrm{~nm}$. This is larger than our results in Fig. 13, but its consequence cannot be judged at this time.

\section{EXPERIMENT}

Figure 14 shows the results of a recent experiment writing $1-\mathrm{nm} \mathrm{W}$ dots from a $\mathrm{W}(\mathrm{CO})_{6}$ precursor in a $200-\mathrm{kV}$ STEM on a $30-\mathrm{nm}$ SiN membrane. The image was taken in high-angle annular dark field mode (HAADF) in order to optimize the contrast of the high- $Z$ material W. We checked that no contamination structures were written when the precursor flow was stopped and we found evidence for $\mathrm{W}$ in EELS spectra of the written features. In Fig. 15, we have averaged over the 100 dots of Fig. 14 by fitting a trapezoidal grid to the positions of the dots and then shifting the local images around the grid positions to the origin of the grid. We

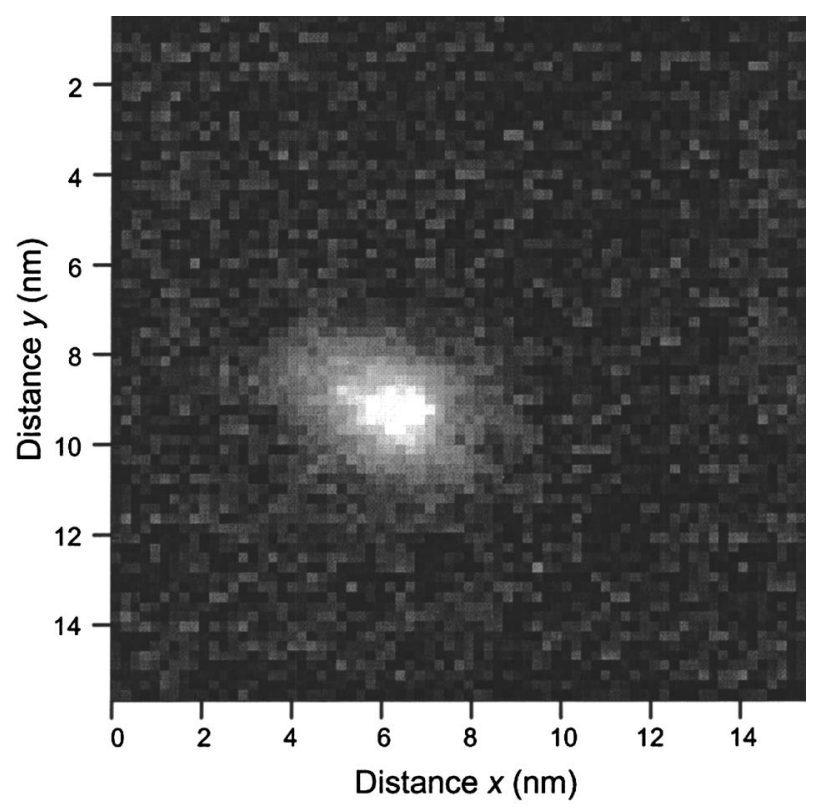

FIG. 15. Averaged profile of the dots in Fig. 14.

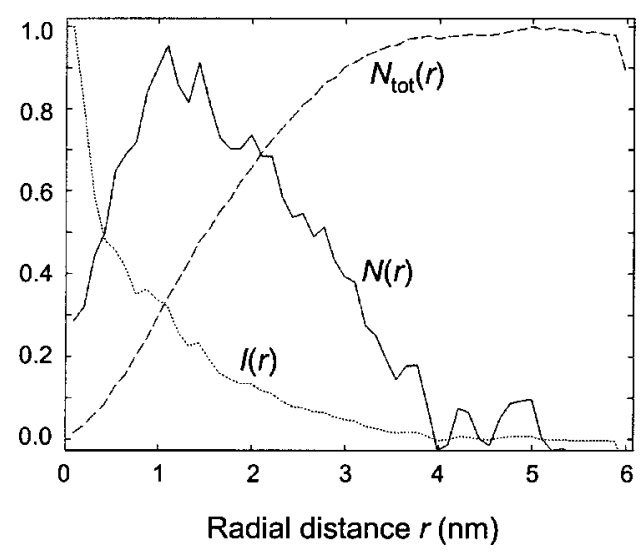

FIG. 16. Radial distribution of image intensity in Fig. 14. Symbols as in Fig. 5. [From Silvis-Cividjian (see Ref. 8).]

had tried to write the dots on a perfectly square grid, but specimen and beam drift during the few seconds of deposition time have displaced the dots from a perfect grid. Fitting to a trapezoid takes out the effects of linear drifts, but not the nonlinear components in the drift nor the arbitrary displacements of the dots. Thus, this procedure will give some widening of the average dot size. We expect that this is also the reason for the slight ellipticity of the profile, although this may also be a streak in the spot with which the dots were written. Figure 16 shows a plot of $I(r)$, effectively the cross section through the dot, obtained by averaging over many different directions through the dot. $N(r)$ is the intensity in a ring of radius $(r, r+d r) . N_{\text {tot }}(r)$ is the integral function of $N(r)$, showing the total amount of $\mathrm{W}$ deposited within a circle of radius $r$.

\section{CONCLUSIONS}

We have described the important steps necessary for the theoretical prediction of EBID lateral resolution given an electron-beam diameter, a gas precursor, and a certain target. The analysis of the spatial resolution has been reduced to the prediction of the shape of the structures deposited under electron-beam irradiation.

Monte Carlo methods and a profile simulator based on cellular automata have been used to model the growth of a singular dot in the spot mode of an electron microscope. In this way we have demonstrated the main role of the secondary electrons in the spatial evolution of structures grown by EBID. The smaller the beam diameter, the more important is the role played by SEs. In contrast with opinions issued until now, the SE spatial information given by classical Monte Carlo programs, used in SEM analysis, is not sufficient to elucidate the role of SEs in EBID. The dot geometry can be evaluated only by taking into account also the energetic distribution of SEs and their molecular dissociation cross section.

The ultimate EBID resolution, calculated for a zerodiameter electron beam and a very thin target $(10 \mathrm{~nm})$, according to our model is around $0.23 \mathrm{~nm}\left(\mathrm{FW}_{50}\right)$. However, the SEs scattered in the freshly grown structure are imposing a larger limit on the spatial resolution of EBID. This is the feasible EBID resolution of around $2 \mathrm{~nm}$ as soon as the 
height of the structure exceeds a few monolayers. The delocalization of inelastic electron scattering at this moment does not impose a fundamental limit to EBID resolution.

The present EBID model can be improved in many ways, for example, by better modeling of SE in the profile simulator. This means the use of a more accurate model for electron scattering in the target, eventually a threedimensional (3D) geometry. In addition, the effect of surface migration of partially decomposed fragments should be studied.

The experimental results, $1-\mathrm{nm} \mathrm{W}$ dots on a $30-\mathrm{nm}$ $\mathrm{Si}_{3} \mathrm{Ni}_{4}$ foil, demonstrate the usefulness of revealing where the limiting factors in EBID resolution are. Five years ago, these results would have seemed impossible.

\section{ACKNOWLEDGMENTS}

We gratefully acknowledge the use of the facilities at the Center for Solid State Science at Arizona State University and the support of Peter A. Crozier.

${ }^{1}$ N. Silvis-Cividjian, C. W. Hagen, L. H. A. Leunissen, and P. Kruit, Microelectron. Eng. 61-62, 693 (2002).

${ }^{2}$ N. Silvis-Cividjian, C. W. Hagen, P. Kruit, M. A. J. van de Stam, and H. B. Groen, Appl. Phys. Lett. 82, 3514 (2003).

${ }^{3}$ Z. Q. Liu, K. Mitsuishi, and K. Furuya, J. Appl. Phys. 96, 3983 (2004).

${ }^{4}$ K. Mitsuishi, M. Shimojo, M. Han, and K. Furuya, Appl. Phys. Lett. 83, 2064 (2003).

${ }^{5}$ P. A. Crozier, J. Tolle, J. Kouvetakis, and C. Ritter, Appl. Phys. Lett. 84, 3441 (2004).

${ }^{6}$ U. Hübner, R. Plontke, M. Blume, A. Reinhardt, and H. W. P. Koops, Microelectron. Eng. 57-58, 953 (2001).

${ }^{7}$ K. T. Kohlmann-von Platen, J. Chlebek, M. Weiss, K. Reimer, H. Oertel, and W. H. Brünger, J. Vac. Sci. Technol. B 11, 2219 (1993).

${ }^{8}$ N. Silvis-Cividjian, Ph.D. thesis, Delft University of Technology, 2002

${ }^{9}$ R. R. Kunz and T. M. Mayer, J. Vac. Sci. Technol. B 5, 427 (1987).

${ }^{10}$ P. C. Hoyle, J. R. A. Cleaver, and H. Ahmed, J. Vac. Sci. Technol. B 14, 662 (1996).

${ }^{11}$ H. W. P. Koops, A. Kaya, and M. Weber, J. Vac. Sci. Technol. B 13, 2400 (1995).

${ }^{12}$ T. E. Allen, R. R. Kunz, and T. M. Mayer, J. Vac. Sci. Technol. B 6, 2057 (1988).

${ }^{13}$ L. G. Christophorou, J. K. Olthoff, and M. V. V. S. Rao, J. Phys. Chem. Ref. Data 25, 1341 (1996).
${ }^{14}$ P. de Jager, Ph.D. thesis, Delft University of Technology, 1997.

${ }^{15}$ A. D. Dubner and A. Wagner, J. Vac. Sci. Technol. B 7, 1950 (1989).

${ }^{16}$ N. Yoshimura, H. Hirano, and T. Etoh, Vacuum 33, 391 (1983).

${ }^{17}$ D. A. Alman, D. N. Ruzic, and J. N. Brooks, Phys. Plasmas 7, 1421 (2000).

${ }^{18}$ K. Uesugi and T. Yao, Ultramicroscopy 42-44, 1443 (1992).

${ }^{19}$ D. C. Joy, Monte Carlo Modeling for Electron Microscopy and Microanalysis (Oxford University Press, New York, 1995).

${ }^{20}$ R. Gauvin and D. Drouin, Scanning 15, 140 (1993).

${ }^{21}$ Z. J. Ding and R. Shimizu, in Proceedings of Second Conference Int. Union Microbeam Analysis Societies, Hawaii, July 2000, Inst. Phys. Conf. Ser. No. 165 (IOP Publishing Ltd., London 2000), pp. 279-280.

${ }^{22}$ M. S. Chung and T. E. Everhart, J. Appl. Phys. 45, 707 (1977).

${ }^{23}$ T. Koshikawa and R. Shimizu, J. Phys. D 7, 1303 (1974).

${ }^{24}$ Z. J. Ding and R. Shimizu, J. Microsc. 154, 193 (1989).

${ }^{25}$ H. Hiroshima and M. Komuro, Jpn. J. Appl. Phys., Part 1 36, 7686 (1997).

${ }^{26}$ L. Reimer, Scanning Electron Microscopy (Springer, Berlin, 1990).

${ }^{27}$ D. Adalsteinsson and J. A. Sethian, J. Comput. Phys. 120, 128 (1995).

${ }^{28}$ A. R. Neureuther, C. H. Ting, and C. Y. Liu, IEEE Trans. Electron Devices ED-27, 1449 (1980)

${ }^{29}$ G. Y. Vichniac, Physica D 10, 96 (1984).

${ }^{30} \mathrm{~S}$. Wolfram, Theory and Applications of Cellular Automata (World Scientific, Singapore, 1986).

${ }^{31}$ D. C. Joy, Ultramicroscopy 37, 216 (1991).

${ }^{32}$ G. Han and F. Cerrina, J. Vac. Sci. Technol. B 18, 3297 (2000).

${ }^{33}$ R. H. Ritchie and A. Howie, Philos. Mag. A 44, 931 (1981).

${ }^{34}$ C. Colliex, Ultramicroscopy 18, 131 (1984).

${ }^{35} \mathrm{H}$. F. Hameka, Advanced Quantum Chemistry Theory of Interactions Between Molecules and Electromagnetic Fields (Addison-Wesley, Reading, Massachusetts, 1965).

${ }^{36}$ R. E. Moss, Advanced Molecular Quantum Mechanics (Chapman and Hall, London, 1973).

${ }^{37}$ J. P. R. Bolton and L. M. B. Brown, Proc. R. Soc. London, Ser. A 428, 2291 (1990).

${ }^{38}$ U. Fano, in Proceedings of Second L. H. Gray Conference, Cambridge, 1969, edited by L. H. Gray and G. E. Adams, Inst. Phys. Conf. Ser. No. 8 (IOP Publishing Ltd., London 1970).

${ }^{39}$ S. J. Pennycook, Ultramicroscopy 26, 239 (1988).

${ }^{40}$ J. D. Jackson, Classical Electrodynamics (Wiley, New York, 1975).

${ }^{41}$ D. A. Muller and J. Silcox, Ultramicroscopy 59, 195 (1995).

${ }^{42}$ J. Drucker, M. R. Scheinfein, J. Liu, and J. K. Weiss, J. Appl. Phys. 74, 7329 (1993).

${ }^{43}$ F. J. Pijper and P. Kruit, Phys. Rev. B 44, 9192 (1991).

${ }^{44}$ V. V. Aristov, N. A. Kislov, and I. I. Khodos, Microsc. Microanal. Microstruct. 3, 313 (1992)

${ }^{45}$ A. Howie, Ultramicroscopy 11, 141 (1983).

${ }^{46}$ T. L. Ferrell and P. M. Echenique, Phys. Rev. Lett. 55, 1526 (1985). 